\title{
An Analysis of Translation Ethics in the Globalization of Chinese literature
}

\author{
Yuanzhen Wu, Shijun Liu \\ Gongqing College Nanchang University, Gongqing, China \\ Gongqing College Nanchang University, Gongqing, China \\ 851301851@qq.com, lindawyz1986@126.com
}

Keywords: Translation ethics; Globalization; Chinese literature

\begin{abstract}
Based on five models of translation ethics put forward by Andrew Chesterman, this paper tries to explore the theoretical basis of the translation of Chinese literature and how to better promote the globalization of it following the notions of translation ethics. Also new forms of translation ethics need to be given further exploration in the information era.
\end{abstract}

\section{Introduction}

Since the building of Babel Tower, translating has become significant in human history, which is described as one of human's basic social behaviors and activities, involving the interrelation between original author, translator and reader. On the other hand, in the field of ethics, it is commonly acknowledged that as long as an activity or behavior is undertaken by human beings, interpersonal relationship emerges and in turn, there exists ethics. Therefore, it follows that translation can be understood to be an ethical activity (Chen Zhendong 2010).

\section{Theoretical basis}

Finnish scholar Andrew Chesterman has made remarkable contribution on the research of translation ethics in which he put forward five ethical models, that of representation, service, communication, norms and professional responsibility, among which the first three are mainly discussed in the following sections.

\subsection{Ethics of representation}

From this perspective, translation, the representation of the original text, is to reflect the meaning of the original text, revealing the faithful principle in the translation standard. Chesterman believes that the ethics of reproduction emphasizes truth and advocates that the translation must reproduce the original text, the author's intention, and the original culture.

\subsection{Ethics of service}

If the ethical study of representation focuses on the relationship between the original text and the translation, the original author and the translator, then the ethical study of the service is the relationship between the translation or the translator and translation user. Structuralism frees the translator from the constraints of the original and the original author, giving the translator full initiative to do the translation so that he or she can translate from the perspective of users of the translation, including readers and translation employers, etc. and serve the responsibilities and purposes of the target users. The translator not only embodies loyalty to the original author, but more importantly, faithful to the user of the translation, so appropriate adjustments will be made to the original text.

\subsection{Ethics of communication}

If the ethics of representation and service indicates the two single-arrow relationships between the translation or the translator to the original or original author and the translation or translator to the 
target user, then the ethical communication is the two-way relationship between the three. It is the exchange of communication between the three, highlighting the role of the translator as a bridge between the other two. The translation not only reproduces the original content and the original author's intention, but also the responsibility and service to the target user. It also takes into account the interpretation of the original text, as well as the effect of the translation in the target language, and the dialogue between the two languages and cultures.

\subsection{Ethics of norms and professional responsibility}

The norm is a requirement for translation behavior or translated text, a measure of translation standards. Chesterman divides the translation specification into expectancy norms and professional norms. The former means the acceptance of the translated text in the target language, and the latter indicates the translator's translation behavior in terms of the acceptance of different readers.

\section{Globalization of Chinese literature}

For a long time, the task of translating Chinese literature has fallen to Chinese translators. However, local translators are not so good at English literature writing, and they lack of in-depth understanding of foreign readers' reading habits and literary publishing market, and consequently it is difficult to obtain the recognition of readers.

For the translation of Chinese culture, people often think that Chinese literature cannot go out because of poor translation. These are the exact limitations and misunderstandings in the interpretation of cultural translation. In professor Xie Tianzhen's view translation is cross-cultural communication. He believes that the goal of translation and translation research is to realize intercultural communication and promote the exchange of different national cultures (Wang Zhiqin, Xie Tianzhen 2013: 26). If just how to faithfully convert the original text is paid attention to, it is difficult for the translation to really enter the cultural system of the target language to achieve effective communication between cultures.

American sinologist Ge Haowen, known as the Western Chief Chinese Literature Translator, has translated Mo Yan's major works and he enjoys a high reputation in the translation industry at home and abroad, who has been described as the midwife of Mo Yan's works in the Western world. In more than 200 works of Mo Yan, Ge Haowen chose ten novels such as Red Sorghum and Life and Death Fatigue as the content of translation, which boosts both the universality of world literature and the heterogeneity of Chinese literature. In the translation strategy of rewriting, Ge Haowen puts Chinese literature in the color of contemporary British and American literature (Bao Xiaoying 2015).

There are reasons that account for Mo Yan's success in the world. His works, translated and introduced in various ways first went abroad through the filming of Red Sorghum; foreign publishers are one of the important ways to promote Mo Yan's works overseas; Mo Yan's works have more mature promotion and agency mechanisms overseas. In addition, participating in international book fairs, joining the international network sales platform such as Amazon, and entering the mainstream newspapers and magazines in the western world are all channels for the translation of Mo Yan's works. As far as the target readers of translation are concerned, Ge Haowen mainly considers the acceptance of the general audience in the West for the translation of Mo Yan's works in the choice of works or the application of translation strategies. He once commented that the most important task of translation is to choose what to translate, rather than how to do the translating. Compared with how to translate, what to translate is the primary issue.

\section{Inspirations of translation ethics}

The ethics of representation reflects the importance of the original text, when the translator is subordinate and the original is greater than everything. The translator's duty is to respect the original author's intention, to be faithful to the original text, to maximize the meaning of the original text in the target language, and the translator itself has no thoughts, emotions or voice. The concept of 
foreignization is the representative of this idea. The translation should have a distinctly exotic flavor and be different from the culture of the target language.

The ethics of service reflects the importance of the target user, that is, the translator's serving object, and in this case the translator is still subordinate. It jumped out of the question of how to be faithful and loyal, and asked why it should be translated and for whom it is translated, that is, the issue of smoothness and elegance. It is also the complexity and variability of service ethics that makes it impossible for machine translation to completely replace humans in translation. The translator's job is to make the translation meet the user's requirements and needs, to be faithful to the recipient of the target language, and to express the original text in the most acceptable way for the user. In such a situation, the translator is given a certain initiative. The concept of domestication is the representative of this idea and the translation should be expressed in this way.

The ethics of communication highlights the importance of the translator who is in a relatively dominant position and emphasizes the reciprocal function of the cultural exchange of translation. The duty is to respect the equal status of the original author, translator and translation user, complete the equal dialogue between the original text and the translation, and achieve the mutual understanding of the author and the users, in which case all three sides have thoughts, emotions, and have the right to speak. Then the translator becomes the messenger of culture. Therefore, the role of the translator is not simply to provide a translation but communicate and coordinate in the relationship between the original author, editor, and sponsor. It must be the intermediate of cultural exchanges, just like business people, negotiators, and diplomats (Pym 2012:120). The degree of reader's recognition of the subject of translation is also particularly important for the acceptance and dissemination of the translation. The higher the recognition, popularity and credibility of the translator, the greater the effect of the translation. (Wang Zhiqing \& Xie Tianzhen 2013:22)

The normative ethics puts objective requirements on the translator from the perspective of the fourth party, and it reflects the normative work of the translator. Its duty is to meet the expectation norms, responsibility norms, communicative norms and relationship norms, and complete the translation work in line with the standards.

The ethics of professional responsibility reflects the translator's professionalism and. the translator is not only an excellent bilingual user, but also an executor who meets the requirements of translation norms, also with the personality of morality, thought and responsibility. This is the most basic professional requirement, and the moral focus here is professional ethics.

\section{Translation ethics in the information era}

In the information era, translation has reached the level of efficiency, speed, sharing, and interaction that cannot be achieved by the traditional translation industry. Internet technology allows translators to freely access social networks, making each participant in the translation community a producer, a translator and a reader where they share resources and translate together (Yin Yan\& Liu Junping 2016). A deep understanding of the ethical foundation of the Internet translation industry will be helpful to understand and grasp the various theoretical and social issues that emerged during the transformation of Internet. Yet, it is still worth further research on the following issues: what is the translation ethics in the information era and what industry ethics does the new form of translation follow? what is the impact it exerts on the globalization of Chinese literature?

\section{References}

[1] Bao Xiaoying, The reception of English Translations of Mo Yan's Oeuvre and the light it sheds on the Globalization of Chinese Literature [J], Chinese Translation,2015(1): 13-17.

[2] Chen Zhendong, Translation ethics: Five Ethical Models of Chesterman, Foreign theoretical development, 2010 (3): 85-88.

[3] Pym, Anthony. On Translator Ethics: Principles for Mediation Between Cultures [M]. Amsterdam/Philadelphia: John Benjamins Publishing Company, 2012. 
[4] Wang Zhiqing \& Xie Tianzhen, The Globalization of Chinese literature: problems and reflections, Monthly Academic Journal, 2013 (2): 21-27.

[5] Yin Yan \& Liu Junping, ethical basis in online translation industry based on Raymond's Open-Source Concept, Journal of Xi' an International Studies University, 2016(3): 111-114. 